\title{
Tests of lepton universality with semitauonic b-quark decays
}

\section{Olivier Leroy ${ }^{* \dagger}$}

Aix Marseille Univ, CNRS/IN2P3, CPPM, Marseille, France

E-mail: Olivier.Leroyein2p3. fr

Tests of Lepton Flavor Universality are presented using ratios of branching fractions $\mathscr{B}(B \rightarrow$ $\left.X_{c} \tau^{+} v_{\tau}\right) / \mathscr{B}\left(B \rightarrow X_{c} \mu^{+} v_{\mu}\right)$, with $X_{c}=D^{*-}$ or $J / \psi$, using the data collected by the LHCb experiment during 2011 and 2012. When combined with the results from the BaBar and Belle experiments, the Standard Model is challenged at the $3.8 \sigma$ level. We also give implications and prospects of these key measurements.

The 39th International Conference on High Energy Physics (ICHEP2018)

4-11 July 2018

Seoul, Korea

* Speaker.

${ }^{\dagger}$ On behalf of the LHCb Collaboration. 
The Standard Model (SM) features Lepton Flavor Universality (LFU), which means that the electroweak couplings to all charged leptons are the same. Hence, the branching ratios to electron, muon and tau leptons should only differ due to their mass. However, a $2.8 \sigma$ tension has been measured already at LEP [1], when comparing the cross-section of $W \rightarrow \tau v_{\tau}$ with respect to the one of $\sigma\left(W \rightarrow e v_{e}\right)+\sigma\left(W \rightarrow \mu v_{\mu}\right)$. This article presented tests of LFU in semitauonic decays using the following ratios:

$$
R\left(X_{c}\right)=\frac{\mathscr{B}\left(B \rightarrow X_{c} \tau^{+} v_{\tau}\right)}{\mathscr{B}\left(B \rightarrow X_{c} \mu^{+} v_{\mu}\right)}
$$

with $X_{c}$ being a $D^{*-}$ or a $J / \psi$ meson and $B$ and a $B^{0}$ or a $B_{c}^{+}$meson ${ }^{1}$. The Standard Model prediction for these ratios are respectively: $R\left(D^{*}\right)=0.258 \pm 0.005$ [2] and $R(J / \psi) \in[0.25,0.28$ ] [3]. These ratios are sensitive to possible New Physics (NP) coupling mainly to the third generation. Feynman diagrams corresponding to $B \rightarrow X_{c} \tau^{+} v_{\tau}$ are shown on Fig. 1: on the left is shown the leading order Standard Model tree-level contribution, while on the right are shown two possible NP contributions: Lepto-Quark [4] and Charged Higgs [5]. The ratios are measured using data collected by the LHCb experiment [6] during the Run 1 at a center-of-mass energy of 7 and $8 \mathrm{TeV}$ in 2011 and 2012, respectively. The $\tau^{+}$lepton is reconstructed either into $\mu^{+} v_{\mu} \bar{v}_{\tau}$ (called muonic) or into $3 \pi\left(\pi^{0}\right) \bar{v}_{\tau}$ (called hadronic) mode.
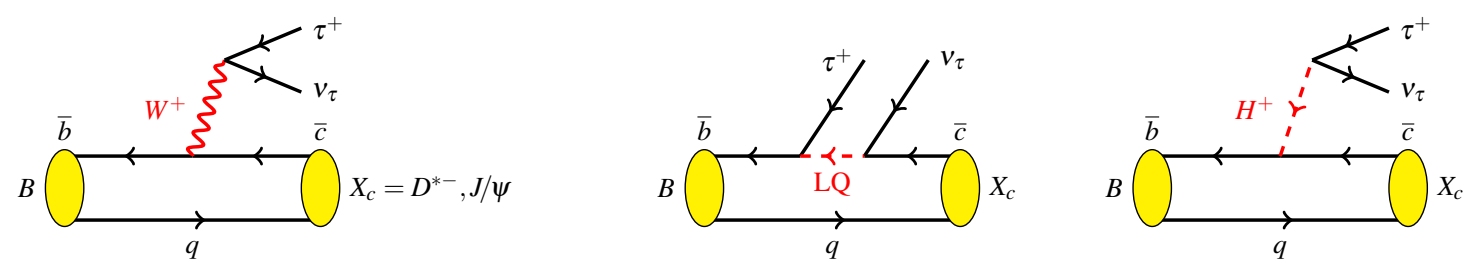

Figure 1: Leading order Feynman diagram for the transition $B \rightarrow X_{c} \tau^{+} \nu_{\tau}$ in the Standard Model on the left and for two possible NP models on the right: Lepto-Quark and Charged Higgs.

For the $R\left(D^{*}\right)$ muonic measurement [7], the normalization mode has the same visible final state as the signal. Signal and normalization are separated by means of a 3D template fit to variables evaluated in the $B$ rest frame: missing mass squared $\left(m_{\text {miss }}^{2}\right)$ muon energy $\left(E_{\mu}^{*}\right)$ and squared 4-momentum transfer to the di-lepton system $\left(q^{2}\right)$. The background and signal shapes are extracted from control samples and simulations validated against data. The result is $R\left(D^{*}\right)=$ $0.336 \pm 0.027$ (stat) \pm 0.030 (syst), $1.9 \sigma$ above the Standard Model expectation. The dominant systematic uncertainty is the size of the simulation samples and it will be improved in the next iteration of the analysis.

For the $R\left(D^{*}\right)$ hadronic $[8,9]$, we measure:

$$
\mathscr{K}\left(D^{*}\right)=\frac{\mathscr{B}\left(B^{0} \rightarrow D^{*-} \tau^{+} v_{\tau}\right)}{\mathscr{B}\left(B^{0} \rightarrow D^{*-} 3 \pi\right)}=\frac{N_{D^{*} \tau v_{\tau}}}{N_{D^{*} 3 \pi}} \times \frac{\varepsilon_{D^{*} 3 \pi}}{\varepsilon_{D^{*} \tau v_{\tau}}} \times \frac{1}{\mathscr{B}\left(\tau^{+} \rightarrow 3 \pi\left(\pi^{0}\right) \bar{v}_{\tau}\right)},
$$

where $N_{X}$ is the number of events of category $X$ and $\varepsilon_{X}$, the efficiency to select those. The $\tau^{+}$is reconstructed into $\pi^{+} \pi^{-} \pi^{+} \bar{v}_{\tau}$, which allows for the $\tau^{+}$vertex to be reconstructed. The normal-

\footnotetext{
${ }^{1}$ Though only $R\left(D^{*-}\right)$ has been measured so far in LHCb, we use the notation " $R\left(D^{*}\right)$ " instead in the rest of this article, asuming, as the b-factories did, that $R\left(D^{*-}\right)=R\left(D^{* 0}\right) \equiv R\left(D^{*}\right)$
} 
ization mode is chosen to have the same final state as the signal. Since there is no charged lepton in these final state, there is no background from the abundant $B^{0} \rightarrow D^{*-} \mu^{+} v_{\mu} X$ decays. $N_{D^{*} 3 \pi}$ is extracted from an un-binned likelihood fit to $D^{*-} 3 \pi$ invariant mass, while $N_{D^{*} \tau v_{\tau}}$ is obtained from a 3D template fit to the decay time of the $\tau$, the $q^{2}$, and a Boost Decision Tree (BDT) variable used to reject the background. Finally, $R\left(D^{*}\right) \equiv \mathscr{K}\left(D^{*}\right) \times \frac{\mathscr{B}\left(B^{0} \rightarrow D^{*-} 3 \pi\right)}{\mathscr{B}\left(B^{0} \rightarrow D^{*-} \mu^{+} v_{\mu}\right)}$. The most abundant background is $b$-hadron decaying to $D^{*-} 3 \pi X$ (which has a branching ratio $\sim 100 \times$ larger than the signal one) is suppressed by requiring the $\tau^{+}$vertex to be downstream with respect to the $B$ vertex along the beam direction. The remaining background is due to doubly charmed decays with non-negligible lifetime. It is rejected with a BDT using in particular the resonant structures of the $\tau^{+} \rightarrow 3 \pi \bar{v}_{v}$ and $D_{s}^{+} \rightarrow 3 \pi X$ decays. The result is $R\left(D^{*}\right)=0.291 \pm 0.019$ (stat) \pm 0.026 (syst) \pm 0.013 (ext), where the third uncertainty originates from the limited knowledge of the branching fraction of the normalization mode. It is $0.9 \sigma$ above the Standard Model expectation. The dominant systematic uncertainty is the size of simulation sample and it will be improved in the next iteration of the analysis.

The ratio $R(J / \psi)$ is analogous of $R\left(D^{*}\right)$, using $B_{c}^{+}$meson [10]. It is defined by: $R(J / \psi)=$ $\frac{\mathscr{B}\left(B_{c}^{+} \rightarrow J / \psi \tau^{+} v_{\tau}\right)}{\mathscr{B}\left(B_{c}^{+} \rightarrow J / \psi \mu^{+} v_{\mu}\right)}, \tau^{+} \rightarrow \mu^{+} v_{\mu} \bar{v}_{\tau}$. The $B_{c}^{+}$has a low production rate and a short lifetime, but the $B_{c}^{+} \rightarrow J / \psi \tau^{+} v_{\tau}$ mode benefit from a clear $J / \psi$ peak that facilitates the reconstruction. Similarly to the $R\left(D^{*}\right)$ analysis, $m_{\text {miss }}^{2}, E_{\mu}^{*}$ and $q^{2}$ are used in a template fit. The information from the $B_{c}^{+}$decay time is added to help. The shapes of various components are represented by a template distribution derived from control samples or simulations validated against data. The main background is $b \rightarrow$ $J / \psi$ accompanied by a mis-identified hadron. The first evidence for the decay $B_{c}^{+} \rightarrow J / \psi \tau^{+} v_{\tau}$ is obtained at $3 \sigma$ level and we measured: $R(J / \psi)=0.71 \pm 0.17$ (stat) \pm 0.18 (syst). The main systematics are due to the form factor and the size of simulation samples.

Three $b \rightarrow c(\mu / \tau) v$ tests of LFU have been performed so far in LHCb: $R\left(D^{*}\right)$ muonic, $R\left(D^{*}\right)$ hadronic and $R(J / \psi)$ muonic. The LHCb average is: $R\left(D^{*}\right)=0.310 \pm 0.0155$ (stat) \pm 0.0219 (syst). It is $1.9 \sigma$ above the SM prediction. When combining with the results from the BaBar and Belle experiments, as well as using the $R(D)$ measurement, the tension with respect to the Standard Model is $3.8 \sigma$ [2], as indicated on Fig. 2, one the highest nowadays in High Energy Physics. The $2 \sigma$ discrepancy observed in $B_{c}^{+} \rightarrow J / \psi \tau^{+} v$ goes in the same direction. The measurements presented were performed using only data collected during the Run 1 period. The Run 2 will bring a factor 5 in statistics. The Run 3 and followings will improve even more these results. Many systematics will be reduced with larger samples. Others will benefit from BESIII and Belle II measurements. Many more analyses will be performed using $b \rightarrow c \tau v$ transitions: $R\left(D^{+}\right), R\left(D^{0}\right)$, $R\left(D_{s}^{(*)-}\right), R\left(\Lambda_{c}^{(*)}\right), \ldots$ and $b \rightarrow u \tau v$ transitions: $R\left(\Lambda_{b}^{0} \rightarrow p \tau v\right), R(B \rightarrow p p \tau v), \ldots$ Interest will then shift towards new observables beyond the branching fraction ratios, for example angular analysis to determine spin structure of potential New Physics. We have a truly exciting work-plan in the coming years, with crucial interplay between experiments and theory.

\section{References}

[1] ALEPH, DELPHI, L3, OPAL, LEP Electroweak, Schael, S. and others, "Electroweak Measurements in Electron-Positron Collisions", Phys. Rept.", 532, 2013, 119-244. 


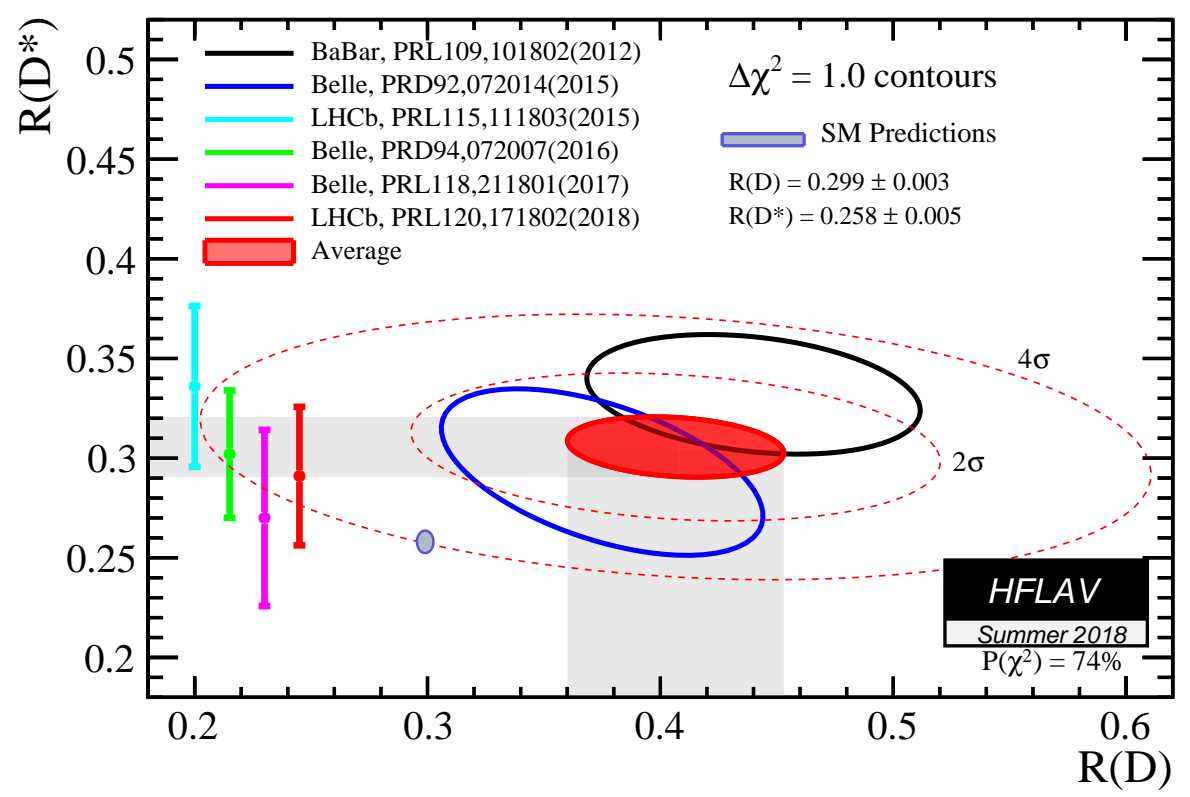

Figure 2: $R(D)$ and $R\left(D^{*}\right)$ measured by the BaBar, Belle and LHCb experiments. The world average value is indicated by the red ellipse while blue ellipse shows the Standard Model expectation [2].

[2] Heavy Flavor Averaging Group (HFAG), Y. Amhis et al. "Averages of b-hadron, c-hadron, and tau-lepton properties as of summer 2016”, Eur. Phys. J. C77 (2017) 895.

[3] A. Anisimov et al., Phys. Lett. B452, 1999 129-136. Kiselev, V.V., hep-ph/0211021. M. A. Ivanov et al., Phys. Rev. D71 094006, 2005. E. Hernández et al., Phys. Rev. D74 074008, 2006.

[4] W. Buchmüller et al., "Leptoquarks in lepton quark collisions", Phys. Lett. B191, 1987, 442-448. "S. Davidson et al., "Model independent constraints on leptoquarks from rare processes", Z. Phys. C61, 1994, 613-644.

[5] M. Tanaka, "Charged Higgs effects on exclusive semitauonic decays" Z. Phys. C67 1995, 321-326.

[6] The LHCb Collaboration, "The LHCb Detector at the LHC", JINST 3 S08005 (2008).

[7] R. Aaij et al. [LHCb Collaboration], "Measurement of the ratio of branching fractions $\mathscr{B}\left(\bar{B}^{0} \rightarrow D^{*+} \tau^{-} \bar{v}_{\tau}\right) / \mathscr{B}\left(\bar{B}^{0} \rightarrow D^{*+} \mu^{-} \bar{v}_{\mu}\right)$," Phys. Rev. Lett. 115 (2015) no.11, 111803 Erratum: [Phys. Rev. Lett. 115 (2015) no.15, 159901].

[8] R. Aaij et al. [LHCb Collaboration], "Measurement of the ratio of the $B^{0} \rightarrow D^{*-} \tau^{+} v_{\tau}$ and $B^{0} \rightarrow D^{*-} \mu^{+} v_{\mu}$ branching fractions using three-prong $\tau$-lepton decays," Phys. Rev. Lett. 120, no. 17, $171802(2018)$.

[9] R. Aaij et al. [LHCb Collaboration], "Test of Lepton Flavor Universality by the measurement of the $B^{0} \rightarrow D^{*-} \tau^{+} v_{\tau}$ branching fraction using three-prong $\tau$ decays," Phys. Rev. D 97 (2018) no.7, 072013.

[10] R. Aaij et al. [LHCb Collaboration], "Measurement of the ratio of branching fractions $\mathscr{B}\left(B_{c}^{+} \rightarrow J / \psi \tau^{+} v_{\tau}\right) / \mathscr{B}\left(B_{c}^{+} \rightarrow J / \psi \mu^{+} v_{\mu}\right)$," Phys. Rev. Lett. 120 (2018) no.12, 121801. 